\title{
Lesión duodenal en el traumatismo contuso de abdomen. Informe de caso y revisión de la bibliografía
}

\author{
Duodenal injury in blunt abdominal trauma. Case report and literature review
}

Jaime A. Rodríguez-García, Aurora N. Ponce-Escobedo*, David A. Pérez-Salazar,

César A. Sepúlveda-Benavides, Rubén A. Uvalle-Villagómez y Gerardo E. Muñoz-Maldonado

Servicio de Cirugía General, Hospital Universitario Dr. José Eleuterio González, Monterrey, Nuevo León, México

\begin{abstract}
Resumen
Existen pocos casos notificados de lesión de intestino delgado por traumatismo contuso abdominal. Se describen la presentación clínica y el tratamiento quirúrgico de dichas lesiones, un caso clínico de un paciente masculino politraumatizado con lesión duodenal IIID3 según la AAST, objeto de resección de segmento intestinal con anastomosis duodenoduodenal terminoterminal con resultados favorables. La presentación infrecuente de lesiones de intestino delgado por traumatismo contuso puede llevar al clínico a soslayar la necesidad de un interrogatorio intencionado acerca de la cinemática del traumatismo y también de los estudios de imagen complementarios diagnósticos debido a la falta de sospecha clínica. Es importante analizar el contexto del paciente para valorar la necesidad de profundizar en estudios diagnósticos y optimizar el tratamiento.
\end{abstract}

PALABRAS CLAVE: Traumatismo. Traumatismo duodenal. Traumatismo contuso. Anastomosis. Lesión. Duodeno.

\begin{abstract}
There are few reported cases of small bowel injury due to blunt abdominal trauma. We describe the clinical presentation and surgical management of these lesions. This is the clinical case of a polytraumatized male with a duodenal injury IIID3 according to AAST, who underwent resection of the intestinal segment with duodeno-duodenum anastomosis with favorable results. The infrequent presentation of injuries to the small intestine due to blunt trauma may lead the clinician to overlook the need for intentional interrogation about the kinematics of the trauma, while at the same time neglecting the taking of complementary diagnostic imaging studies, this because of a lack of clinical suspicion. It is important to analyze the patient's context, which will allow us to assess the need to delve into diagnostic studies in order to optimize their treatment.
\end{abstract}

KEY WORDS: Traumatism. Duodenal trauma. Blunt trauma. Anastomosis. Injury. Duodenum.

\section{Introducción}

Las lesiones duodenales se presentan en menos del $5 \%$ de los pacientes con traumatismo abdominal en general'. En una revisión de la bibliografía de 24 series publicadas durante 46 años (1968-2014) se identificaron 1760 casos de lesión duodenal, de los cuales
1400 (80\%) ocurrieron como consecuencia de traumatismo penetrante, mientras que $360(20 \%)$ se debieron a traumatismo contuso (la causa fue en $85 \%$ los accidentes automovilísticos ${ }^{2}$ ). Su incidencia es mayor en el sexo masculino con una relación de 5 a 1 en comparación con el sexo femenino debido a la mayor incidencia de traumatismo contuso de abdomen en

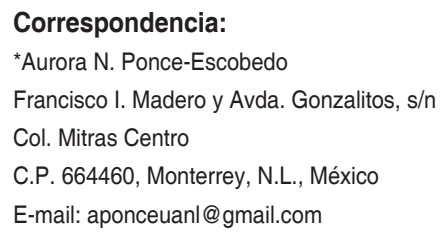

Cir Cir. 2019;87(S1):53-57

Contents available at PubMed www.cirugiaycirujanos.com 
hombres. En la actualidad, la principal causa de muerte en pacientes entre la tercera y la cuarta décadas es el traumatismo, en virtud del aumento de situaciones de agresión personal y accidentes automovilísticos. ${ }^{2-4}$

La disposición anatómica de esta estructura explica la baja incidencia de lesión en el traumatismo abdominal contuso y son un reto para el cirujano el diagnóstico y el tratamiento ${ }^{5}$. El principal mecanismo lesivo consiste en el aplastamiento causado por el volante, o el manubrio de una bicicleta, contra los cuerpos vertebrales, lo que produce laceración directa o elevación de la presión intraluminal que ocasiona la rotura de la pared duodenal ${ }^{4}$. Otra causa menos común es la desaceleración. Estas lesiones se presentan sobre todo en la tercera y cuarta porciones del duodeno, la unión de la parte retroperitoneal e intraperitoneal. Una demora en su identificación implica una intervención quirúrgica más compleja y eleva de forma notable la morbimortalidad ${ }^{6,7}$. Dada su estrecha proximidad con otras vísceras y estructuras vasculares, es común encontrar lesiones relacionadas en particular con lesiones penetrantes. Las lesiones duodenales aisladas en el traumatismo contuso son excepciona$\operatorname{les}^{8}$. Las lesiones secundarias a un traumatismo duodenal se identifican en el $70 \%$ de las ocasiones ${ }^{9}$. En el caso siguiente se describe un episodio excepcional e infrecuente de una lesión duodenal aislada.

Las nuevas modalidades de imagen como la tomografía computarizada (TC) han demostrado una mayor sensibilidad y especificidad para demostrar lesiones duodenales en el traumatismo abdominal cerrado y delinean signos directos e indirectos que ayudan a establecer un tratamiento quirúrgico temprano ${ }^{10}$.

No existe un consenso que determine la conducta a seguir ante una lesión duodenal, aunque se han descrito desde reparaciones primarias hasta exclusiones pilóricas con gastroyeyunostomías y procedimiento de Whipple en lesiones más complejas ${ }^{11,12}$. En todo caso, el tratamiento debe individualizarse.

\section{Caso clínico}

Paciente masculino de 43 años de edad que sufre un accidente automovilístico con colisión frontal; ingresa a la sala de choque-traumatismo consciente y estable en términos hemodinámicos; se decide realizar FAST que revela escaso líquido libre en la fosa esplenorrenal. Dada la estabilidad del paciente se realiza tomografía contrastada de abdomen que identifica hematoma hepático en segmento $V$ relacionado con escaso líquido libre perihepático, lesión renal de

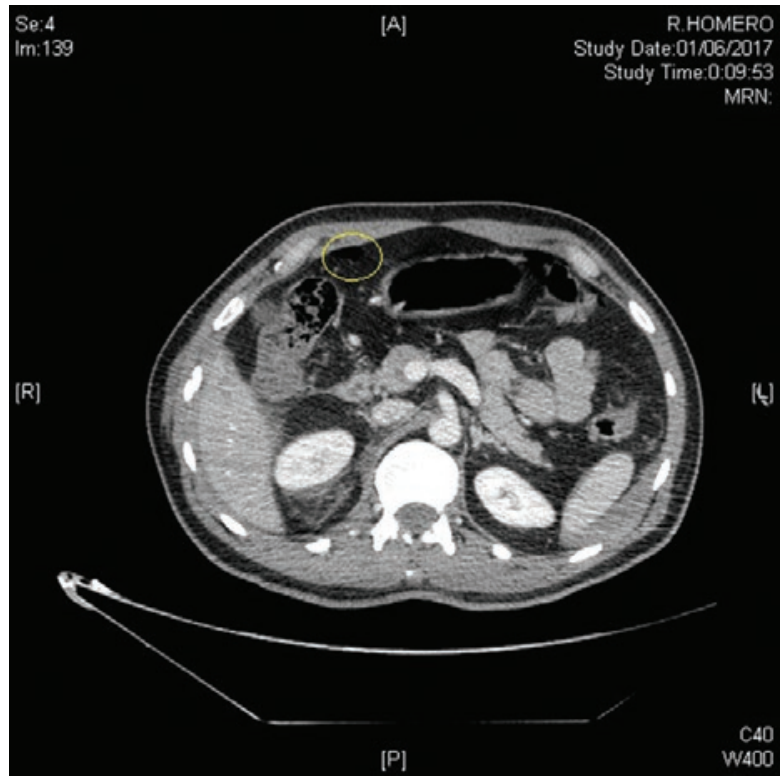

Figura 1. Evidencia de aire libre en la cavidad abdominal.

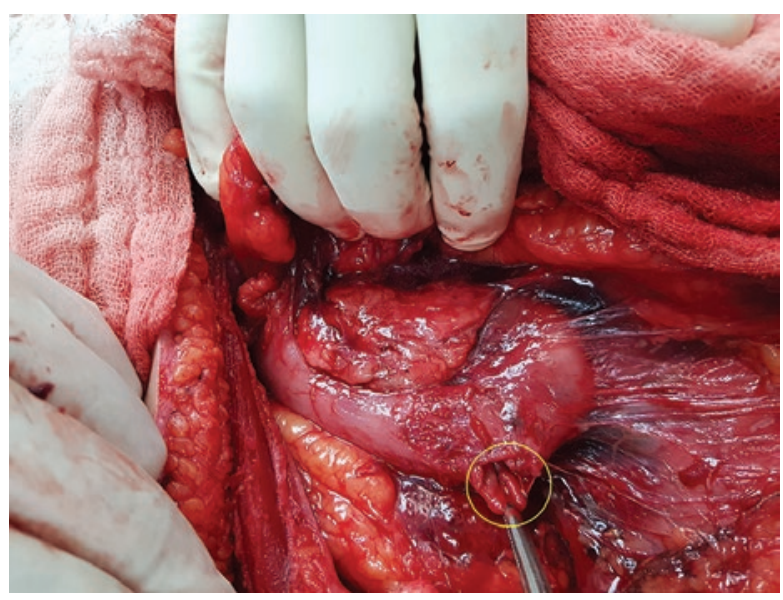

Figura 2. Lesión de grado III en la tercera porción del duodeno.

grado III e imágenes con densidad de aire en la cavidad abdominal y retroperitoneo (Fig. 1). Se efectúa laparotomía exploradora que muestra $100 \mathrm{~cm}^{3}$ de hemoperitoneo, además de laceración de mesocolon derecho con pérdida de la vasculatura del colon ascendente; se realiza maniobra de Kocher que revela una lesión de grado $\mathrm{II}^{13}$ de la tercera porción del duodeno (Fig. 2), además de segmento de íleon a $40 \mathrm{~cm}$ de la válvula ileocecal con cambios isquémicos debido al desgarro del mesenterio (lesión intestinal de grado $\mathrm{V}$ ). Se practican una resección del segmento afectado de duodeno y una anastomosis duodenoduodenal terminoterminal con puntos de Connel-Mayo. Se reseca un segmento de $10 \mathrm{~cm}$ con cambios isquémicos a $40 \mathrm{~cm}$ de la válvula ileocecal y se anastomosa con la misma técnica, además de hemicolectomía derecha del segmento desvascularizado con 
íleo transverso y anastomosis terminolateral y por último una gastrostomía y yeyunostomía tipo Witzel. Durante la evolución postoperatoria, el paciente permanece estable, con nutrición parenteral en los primeros cinco días e inicio de nutrición por yeyunostomía al sexto día, sin datos de alteraciones hidroelectrolíticas o leucocitosis. Se solicita amilasa sérica de control que se encuentra dentro de valores normales. Durante el décimo día postoperatorio se obtiene TC de abdomen con contraste intravenoso e intraluminal para evaluar anastomosis y se informa paso adecuado de contraste sin presencia de colecciones intraabdominales, por lo que se inicia dieta enteral que se tolera. El paciente continúa su evoluciona favorable por lo que se decide el egreso hospitalario al día $13^{\circ}$ del postoperatorio con seguimiento ambulatorio por consulta externa.

\section{Discusión}

En virtud de la escasa frecuencia de lesiones duodenales en el traumatismo abdominal contuso, no es raro que éstas pasen inadvertidas en la valoración inicial, lo cual representa un retraso diagnóstico ${ }^{14,15} \mathrm{y}$, en consecuencia, es necesaria una alta sospecha de lesión; no deben perderse de vista la cinemática del traumatismo y las situaciones de la escena del traumatismo, por ejemplo el tipo de impacto, tipo de caída, altura de la caída, tipo de vehículo o velocidad de impacto, para poder determinar una lesión por aceleración o desaceleración o bien por fuerza directa contra la pared abdominal ${ }^{2}$. La exploración física proporciona datos inespecíficos de un abdomen agudo, como dolor abdominal, signo de rebote positivo y ausencia de ruidos peristálticos. Sin embargo, la presencia de estos signos durante la exploración física no es específica o suficiente para precisar la localización exacta del órgano lesionado. El diagnostico debe apoyarse con estudios de imagen complementarios, siempre que el estado hemodinámico del paciente lo permita. Los estudios como la tomografía computarizada de abdomen, con contraste intravenoso e intraluminal ${ }^{16}$, proporcionan una visión de los órganos retroperitoneales y datos sensibles de lesión, además de una información detallada de los órganos afectados, como el bazo o el hígado. El tratamiento quirúrgico de la lesión al duodeno depende del estado del paciente y la porción del duodeno afectada. Cuando tiene lugar una lesión duodenal, el sitio anatómico de mayor frecuencia lesionado es la segunda porción (36\% de las veces), seguido de la tercera porción
Tabla 1. Clasificación de las lesiones de vísceras huecas y sólidas según la American Association for the Surgery of Trauma

\begin{tabular}{|c|c|c|}
\hline Grado & Lesión & Descripción \\
\hline I & $\begin{array}{l}\text { Hematoma } \\
\text { Laceración }\end{array}$ & $\begin{array}{l}\text { Afectación de una sola porción duodenal } \\
\text { Laceración parcial de la pared, sin } \\
\text { perforación }\end{array}$ \\
\hline$\|$ & $\begin{array}{l}\text { Hematoma } \\
\text { Laceración }\end{array}$ & $\begin{array}{l}\text { Afectación de más de una porción } \\
\text { Alteración de menos del } 50 \% \text { de la } \\
\text { circunferencia duodenal }\end{array}$ \\
\hline III & Laceración & $\begin{array}{l}\text { Alteración del } 50 \text { a } 75 \% \text { de la } \\
\text { circunferencia de la segunda porción } \\
\text { duodenal } \\
\text { Alteración de } 50 \text { a } 100 \% \text { de la } \\
\text { circunferencia de la primera, tercera y } \\
\text { cuarta porciones duodenales }\end{array}$ \\
\hline IV & Laceración & $\begin{array}{l}\text { Alteración de más del } 75 \% \text { de la } \\
\text { circunferencia de la segunda porción } \\
\text { duodenal } \\
\text { Afectación del ámpula duodenal o el } \\
\text { colédoco distal }\end{array}$ \\
\hline V & $\begin{array}{l}\text { Laceración } \\
\text { Vascular }\end{array}$ & $\begin{array}{l}\text { Alteración masiva del complejo } \\
\text { duodenopancreático Desvascularización } \\
\text { del duodeno }\end{array}$ \\
\hline
\end{tabular}

(18\%): la porción menos lesionada es la primera, lo cual podría explicarse por su localización anatómica particular ${ }^{2}$. El tratamiento del paciente con un abdomen agudo por traumatismo termina con el traslado al paciente a la sala de operaciones para efectuar una laparotomía exploradora. Se debe realizar un control de daños y conceder prioridad a las estructuras vasculares, lesiones adjuntas frecuentes en este tipo de traumatismo ${ }^{17,18}$. Con posterioridad deben valorarse los órganos sólidos y, por último, las vísceras huecas. Durante la laparotomía es necesario realizar una revisión detallada e intencionada de las cuatro porciones del intestino delgado. Hallazgos como líquido libre, líquido con características biliares y hematomas periduodenales son indicadores de lesión del intestino delgado². El grado de lesión del duodeno y el sitio afectado determinan el tratamiento quirúrgico. Los grados de la lesión duodenal se establecen de acuerdo con la American Association for the Surgery of Truma (AAST), que clasifica las lesiones de vísceras huecas y sólidas en los grados I al V, como se muestra en la Tabla $1^{13}$.

Existen distintos tipos de reparación y se basan en los hallazgos del cirujano, el compromiso de la encrucijada biliopancreática, la estabilidad hemodinámica del paciente o el tiempo transcurrido desde la lesión ${ }^{19}$; todos son factores que incrementan la morbimortali$\mathrm{dad}^{20}$. Si es posible, un cierre primario en sentido 
transversal para evitar la estenosis puede ser suficiente, pero en una lesión compleja acompañada de lesión pancreática o lesión vascular podría estar indicada una duodenopancreatectomía. Stone y Fabián en 1979 introdujeron el uso del tubo de duodenostomía como triple ostomía (gastrostomía, duodenostomía y yeyunostomía $)^{21}$ y muchos cirujanos aún lo utilizan para descomprimir el duodeno a pesar de que numerosos estudios no han mostrado beneficio y han revelado que eleva la morbilidad. Sin embargo, existe una amplia variedad de técnicas quirúrgicas a considerar; en este caso se trata de una lesión compleja de la tercera porción de duodeno en la cual se optó por efectuar una resección de la lesión con anastomosis duodenal terminoterminal con sutura continua, puntos de Connel y Mayo y, como protección de la línea de sutura, se derivó el contenido gástrico por medio de gastrostomía de Witzel; asimismo, en la misma intervención se realizó una yeyunostomía con la finalidad de iniciar una dieta enteral distal al sitio de lesión sin una exclusión pilórica o un procedimiento mayor con menor morbilidad y menor tiempo quirúrgico. Entre las técnicas descritas figuran las siguientes: duodenorrafia primaria, anastomosis duodenal, diverticulización duodenal, anastomosis duodenoyeyunal, exclusión pilórica, etc. El principal objeto de estudio entre el discernimiento de las técnicas propuestas es, sin duda alguna, el manejo postoperatorio, el riesgo de fuga anastomótica o de fuga de una reparación primaria ${ }^{22}$. Por lo regular son preferibles las reparaciones primarias en las lesiones del duodeno de los grados I y II en los que la serosa se afronta mediante puntos de sutura de Lembert; en los casos de lesiones de grado II se realiza el cierre primario sin tensión en una o dos capas ${ }^{23}$. En algunos casos, cuando el defecto traumático es mayor de $3 \mathrm{~cm}$, se debe considerar una recesión del segmento afectado con anastomosis duodenoduodenal debido al riesgo de estenosis del sitio reparado. En casos muy particulares, en los que la segunda porción del duodeno esté comprometida, deben considerarse otras técnicas más complejas según sea el grado de la lesión (III ○ V), desde la colocación de prótesis, drenaje y anastomosis enterobiliares hasta los procedimiento de Whipple, siempre bajo el contexto de un paciente hemodinámicamente estable en el transoperatorio ${ }^{23}$. Existe aún controversia acerca de la necesidad de crear una exclusión pilórica en los pacientes sometidos a una derivación de secreciones gástricas del sitio reparado; sin embargo, el uso de esta técnica ha mostrado un aumento de úlceras marginales a lo largo de la anastomosis gastroyeyunal adjunta ${ }^{23}$.

La mortalidad de una lesión duodenal varía de manera amplia en las publicaciones médicas, desde 5 hasta $30 \%$; esta gran variación en porcentaje se debe a la descripción de las dos causas principales de la mortalidad ${ }^{3,22}$. La primera causa considerada como muerte temprana se vincula con las lesiones vasculares acompañantes, con diagnóstico y tratamiento quirúrgico tardíos; la mortalidad tardía se debe al desarrollo de sepsis abdominal por causa de un diagnóstico tardío o complicaciones en el postoperatorio como la fuga de una reparación o una anastomosis ${ }^{3,22}$.

\section{Conclusiones}

Es importante para el clínico mantener una alta sospecha de lesión duodenal en pacientes con traumatismo contuso de abdomen. La cinemática del traumatismo es de gran ayuda para elevar la sospecha, por lo que no debe soslayarse y de esta manera es necesario buscar de manera intencionada datos que lleven a identificar de manera temprana una lesión duodenal. Para reforzar o confirmar la sospecha deben indicarse estudios de imagen como la tomografía computarizada con contraste intravenoso e intraluminal, en casos de estabilidad hemodinámica.

Respecto del tratamiento oportuno: cuanto más rápido se establezca un tratamiento quirúrgico mejores serán los resultados. Ante la duda es mejor realizar la exploración quirúrgica con incisión en línea media y movilizar el duodeno con maniobras de Kocher y Cattel-Brash para reconocer todas las porciones duodenales y siempre con el principio del control de daños en mente.

El comportamiento quirúrgico depende del sitio y tipo de lesión. Es seguro realizar reparaciones primarias siempre y cuando la lesión tenga menos de $24 \mathrm{~h}$ de evolución. El desbridamiento de bordes o la resección del segmento afectado con anastomosis terminoterminal son una opción factible y segura, a condición de que no exista compromiso del ámpula de Vater. Es recomendable la colocación de una vía de alimentación enteral temprana para mantener al paciente con un adecuado apoyo nutricional.

\section{Conflicto de intereses}

Los autores declaran que no existe ningún potencial conflicto de interés relacionado con el artículo descrito. 


\section{Responsabilidades éticas}

Protección de personas y animales. Los autores declaran que para esta investigación no se han realizado experimentos en seres humanos ni en animales.

Confidencialidad de los datos. Los autores declaran que han seguido los protocolos de su centro de trabajo sobre la publicación de datos de pacientes.

Derecho a la privacidad y consentimiento informado. Los autores han obtenido el consentimiento informado de los pacientes y/o sujetos referidos en el artículo. Este documento obra en poder del autor de correspondencia.

\section{Bibliografía}

1. Velmahos GC, Kamel E, Chan LS. Complex repair for the management of duodenal injuries. Am Surg. 1999;65(10):972-975

2. García Santos E, Soto Sánchez A, Verde JM, Marini CP, Asensio JA Petrone P. Duodenal injuries due to trauma: review of the literature. Cirugía Española (English ed. 2015;93(2):68-74. doi:10.1016/j.cireng.2014.12.006

3. Moore EE, Cogbill TH, Malangoni MA, Jurkovich GJ, Champion HR. Scaling system for organ specific injuries. Curr Opin Crit Care. 1996;2(6):450-462. doi:10.1097/00075198-199612000-00008.

4. Instituto Nacional de Estadística y Geografía (México). (2018). Mujeres y Hombres en México 2018. Disponible en: http://cedoc.inmujeres.gob.mx

5. Degiannis E, Boffard K. Duodenal injuries. Br J Surg. 2000:1473-1479.

6. Zhi-ying Y, Tianyi C, Xin L, Xinting S, Wei-song T, Shouxian Z. Technical note. A novel method of pyloric occlusion in the treatment of blunt retroperitoneal duodenal injuries (BRDI) with delayed diagnosis. Injury Extra 2010;41(1):25-28. doi:10.1016/j.injury.2009.11.004.

7. Allen GS, Moore FA, Mehall JR, Duke JH. Delayed diagnosis of blunt duodenal injury: an avoidable complication. J Am Coll Surg. 1997; 7515(98):393-399.
8. Pirozzi C, Di Marco C, Loponte M, Savino G. Total transverse rupture of the duodenum after blunt abdominal trauma. Ann Ital di Chir U6 - ctx_ver=Z3988-2004\&ctx_enc=info\%3Aofi\%2Fenc\%3AUTF-8\&rfr_id=info\%3Asid\%2Fsummon.serialssolutions.com\&rft_val_ $\mathrm{fmt}=$ info\%3Aofi\%2Ffmt\%3Akev\%3Amtx\%3Ajournal\&rft.genre=article \&rft.atitle = Total+transverse +rupture +of +the+duoden. 2014;85(ePub).

9. Garcia AF, Ferrada P, Wolfe L, Duchesne J, Fraga GP. Management of duodenal trauma : a retrospective review from the Panamerican Trauma Society. 2018;(March 2019). doi:10.1097/TA.0000000000002157.

10. Bradley M, Bonds B, Dreizin D. Indirect signs of blunt duodenal injury on computed tomography: Is non-operative management safe? Injury. 2016;47(1):53-58. doi:10.1016/j.injury.2015.10.003.

11. Kasotakis G. Safety of repair for severe duodenal injuries. 2008:7-12. doi:10.1007/s00268-007-9255-4.

12. Ivatury R, Nassoura Z, Simon R, Rodriguez A. Complex duodenal injuries. Surg Clin North. 1996;76(4):1-13.

13. The American Association for the Surgery of Trauma AAST. (2016). Injury Scoring Scale. A Resource for Trauma Care Professionals. Disponible en: http://www.aast.org/library/traumatools/injuryscoringscales.aspx

14. Bhattacharjee $\mathrm{H}$, Misra M, Kumar S, Bansal V. Duodenal perforation following blunt abdominal trauma. J Emerg Trauma Shock. 2011;4:514.

15. Singh B. Combined gastric and duodenal perforation through blunt abdominal Trauma. J Clin Diagnostic Res. 2015;9(1):30-33. doi:10.7860/ JCDR/2015/11206.5499.

16. Carrillo EH, Richardson JD, Miller FB. Evolution in the management of duodenal injuries. J Trauma Acute Care Surg. 1996;40(6).

17. Chen C, Schuster K, Bhattacharya B. Motor vehicle collision patient with simultaneous duodenal transection and thoracic aorta injury: a case report and review of the literature. Case Rep Surg. 2015;2015:519836. doi:10.1155/2015/519836.

18. Pandey S, Niranjan A, Mishra S. Retrospective analysis of duodenal injuries: a comprehensive overview. Saudi J Gastroenterol. 2011;17:142.

19. Lucas CE, Ledgerwood AM. Factors influencing outcome after blunt duodenal injury. J Trauma Acute Care Surg. 1975;15(10).

20. Snyder WH $3^{\text {rd }}$, Weigelt JA, Watkins WL, Bietz DS. The surgical management of duodenal trauma. Precepts based on a review of 247 cases. Arch Surg. 1980;115(4):422-429.

21. Stone $\mathrm{H}$, Harlan MD, Fabian TCMD. Managment of duodenal wounds. J Trauma. 1979;19(5):334-339.

22. Kong V, Bruce JL. Duodenal trauma. SJS 2019. 2019;(January). doi:10.1177/1457496918822620.

23. Sánchez IJM, Manuel L, Núñez G. Alternativas de manejo del trauma duodenal. 2013;35:34-37. 